\title{
Conceptual design of financial ontology
}

\author{
Helena Dudycz \\ Wrocław University of Economics \\ Komandorska Str. 118/120, \\ PL 53-345 Wroclaw, Poland \\ Email: helena.dudycz@ue.wroc.pl
}

\author{
Jerzy Korczak \\ Wrocław University of Economics \\ Komandorska Str. 118/120, \\ PL 53-345 Wroclaw, Poland \\ Email:jerzy.korczak@ue.wroc.pl
}

\begin{abstract}
The article presents the approach to the conceptualization of the financial knowledge used for an Intelligent Dashboard for Managers. The content of the knowledge is focused on essential financial concepts and relationships related to the management of small and medium enterprises (SME). That includes the illustration of the conceptualization of the ontology. The designed ontology was split into six ontologies describing areas of Cash Flow at Risk, Comprehensive Risk Measurement, Early Warning Models, Credit Scoring, Financial Market, and General Financial Knowledge. The examples of the topic map of key financial indicators and their interpretation are given. The results of this research have been implemented in the Business Intelligence system.
\end{abstract}

\section{INTRODUCTION}

$\mathrm{T}$ O make optimal decisions, managers need very useful, adequate and easy to interpret information. They must analyse various economic indicators assessing the financial situation of an enterprise. Data for analyses are usually extracted from different information systems. To interpret a financial indicator, a manager should analyze relations between indicators and economic data which have influence on its value. However, available information systems concentrate mainly on providing information reflecting hierarchic relationships between examined indicators. Decision-makers evaluate semantic associations existing between them. Such an analysis of indicators can potentially ease and shorten the time needed, inter alia, to identify chances of advancement and threats of breakdown related to carrying out an activity. In order to facilitate the process of data analysis, the usage of the ontology is proposed as a model of financial knowledge about the analysis of indicators.

The decision-makers of small and medium enterprises (SMEs), in comparison to managers of big companies, may not have access to all essential strategic information. Usually financial expertise is either not available or too expensive. Big companies have at their disposal strategic consultation and possess standard procedures to solve problems in the case of essential changes in business environment. For financial and personnel reasons most SMEs cannot afford these types of facilities. It should be noted that SMEs operate in a definitely more uncertain and risky environment than big enterprises, because of a complex and dynamic market that has much more important impact on SMEs' financial situation than on big companies [1].

In general, most existing Business Intelligence (BI) and Executive Information Systems (EIS) provide the functionality of data aggregation and visualization. Many reports and papers in this domain underline that decision makers expect new ICT solutions to interactively provide not only relevant and up-to-date information on the financial situation of their companies, but also explanations taking into account the contextual relationships.

Our research concentrates on two essential issues: supporting decision makers in the area of analysis of economic and financial information using solutions for representing the ontology of economic and financial data (for example: topic map) ${ }^{1}$, and using tools for visualization of the semantic network, which is based on an ontology model of the economic knowledge and data from all relevant information systems ${ }^{2}$.

The aim of this article is to present the conceptual design of financial ontology. The structure of the paper is as follows. In the next section, the functional schema of the system is discussed. The main domain areas of financial knowledge are presented and detailed by the topic map of the main financial indicators. Section 3 describes the process of ontology development, in particular the actual design of the ontology. A case study in section 4 illustrates an example of financial ontology conceptualisation. To show the reasoning a case for explanation of financial data is specified. In the conclusion, the future research directions are indicated.

1 This research was supported by the National Research and Development Centre within the Innotech program (track In-Tech), grant agreement no. INNOTECH-K1/IN1/34/153437/NCBR/12. The name of this project was the Intelligent Dashboard for Managers, which was conducted by a consortium led by the Wrocław University of Economics, Poland (the leader: Jerzy Korczak), and the other principal member is the company UNIT4 TETA BI Center. Credit Agricole Polska also participated in the project. The project was realized during the period 2012-2014.

This research was supported by the Polish Ministry of Science and Higher Education (grant N N111 284038 ). This grant was realised by Helena Dudycz during the period 2010-2012. 


\section{II.PROPOSAL WIDEN BUSINESS INTELLIGENCE SYSTEM FUNCTIONALITIES}

The Business Intelligence (BI) system is used for the analysis of all basic areas of an enterprise's activities, such as, e.g., finance and accounting, manufacturing, logistics, marketing, sales, and customer relationships. These applications provide many reports containing valuable information in each statement. Retrieval information from these reports is eased by the use of appropriate forms of its presentation, and of a friendly and easy user interface. Nowadays decision-makers want not only to look at static reports or even ad hoc reports, but also easy-to-use tools to assess goals and key performance indicators to identify any chances of advancement and threats of breakdown. The usefulness of the BI system is not related to the amount of generated information, but to the information which is required at the right moment. These were basic motives for developing and applying a new technology and knowledge representation in the $\mathrm{BI}$ system. In the literature, the development of BI systems towards BI 2.0 (using semantic search) is described (see [2]-[4]). This system is focused on the semantic analysis of data, using data and information from multiple sources (including external sources). One of the main artifacts to create a semantic network is the ontology, because the architecture of BI 2.0 has new components, such as ontologies and service ontologies (see [2]). The ontologies are used to create the necessary knowledge models for defining and explaining functionalities in analytical tools. Using ontologies and semantic networks for a visual interface supporting an information search in the BI system may help to reduce the following weaknesses of management information systems (see [4], [5]):
- lack of support in defining business rules for getting proactive information and support in consulting in the process of decision making;

- lack of a semantic layer describing relations between different economic topics;

- lack of support in presenting the information of different users (employees) and their individual needs;

- difficulty in rapidly modifying existing databases and data warehouses in the case of new analytic requirements. In Figure 1 a functional architecture of the information system is presented, with ontology applications. Various mechanisms can be seen for extracting source data from transactional systems (ETL), its data warehouse, and external sources. However, the available solutions - in particular the standard analyses, reports and analytical statements generated by the system - are complemented by economic and financial knowledge (most importantly ontologies). This enables a dynamic, interactive analysis of key economic and financial indicators. Such architecture concept was used in the project InKoM (a wide review of the issue is presented in: [6], [7]). This solution will significantly extend existing $\mathrm{BI}$ and EIS functionalities.

To support the analysis, SMEs decision makers need economic and financial knowledge. The scope of required knowledge was divided arbitrarily by experts into six selected areas, namely: Cash Flow at Risk, Comprehensive Risk Measurement, Early Warning Models, Credit Scoring, Financial Market, and General Financial Knowledge (Fig. 2). They are described in [6]. Between these fields there are intersections, and some topics belong to two or three areas.

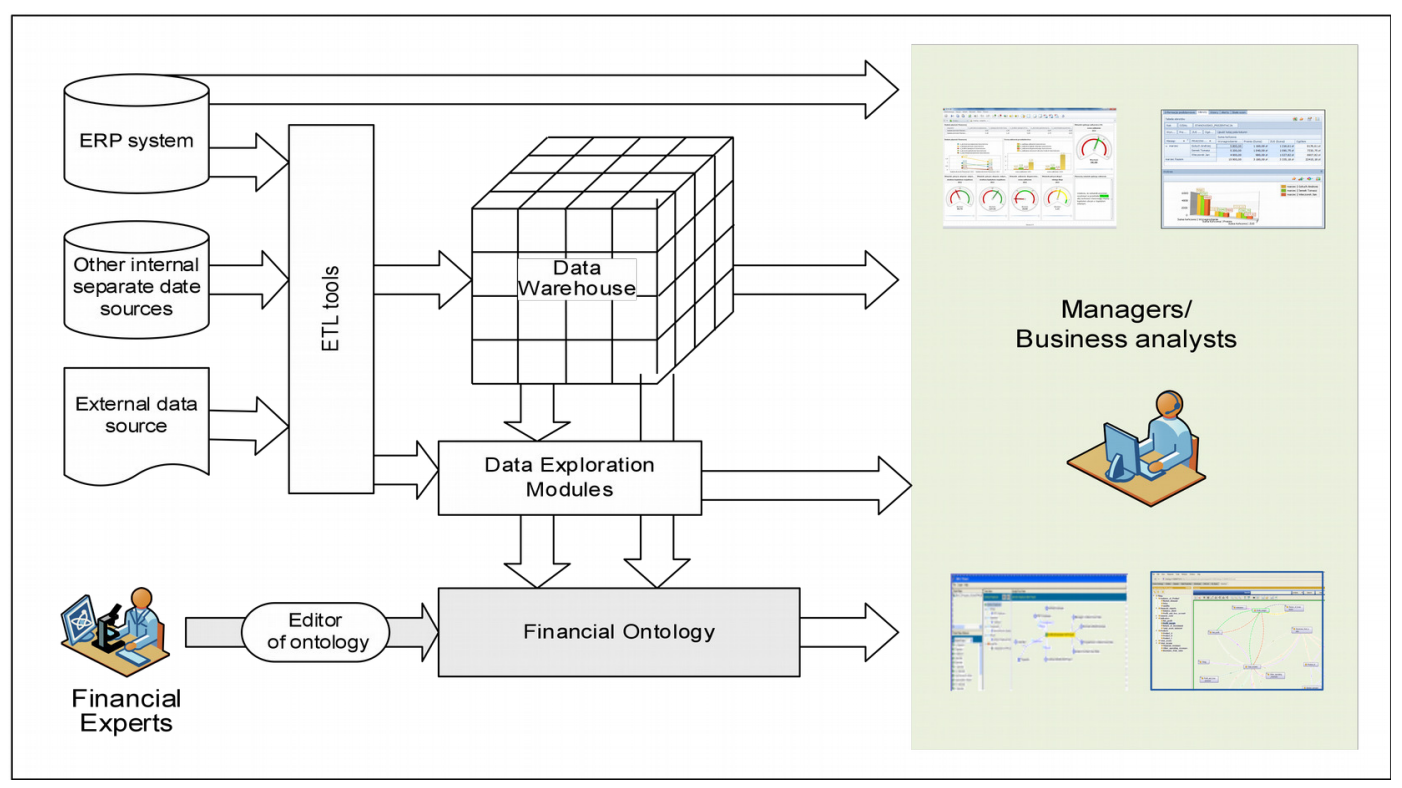

Fig. 1. Functional architecture of information system with ontology applications

Source: based on [1, p. 57]. 
The system that enables semantic information retrieval should be intuitive to use or easy to understand. For managers, the presentation layer is the most critical aspect of a BI system, since it broadly shapes their core understanding of the data displayed [8]. The basic assumption of navigation is that managers should be able to view focus and context areas at the same time to present an overview of the whole knowledge structure [9].

Ontology of financial knowledge is the foundation of creating a semantic network. In our project, special attention was paid to the role of the visualization of a semantic network, which is not only a tool for presenting data, but also provides an interface allowing interactive visual information retrieval (see inter alia [10], [11]). Working from the displayed semantic structure of a built-in ontology of financial and economic knowledge, it is possible to interactively choose analyzed topics or relations, to change the area of presented details, and to obtain relevant source data.

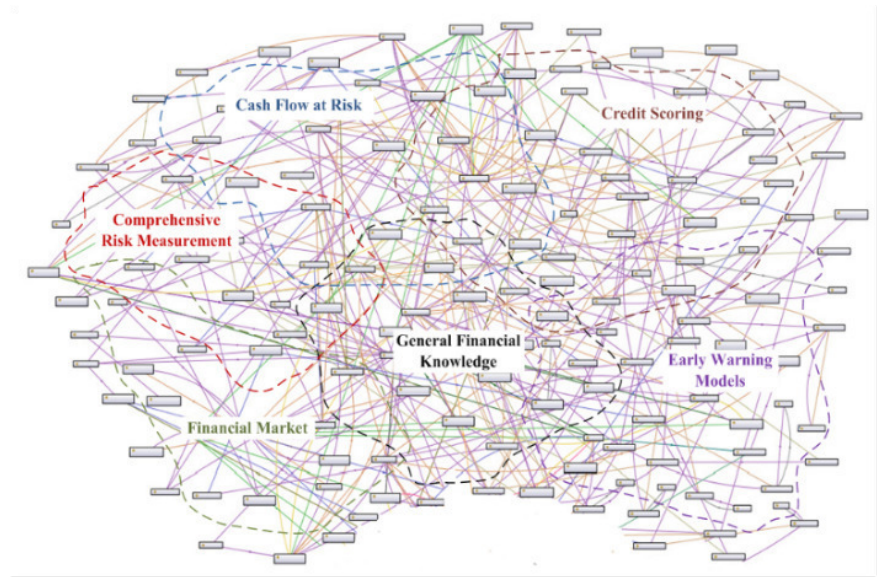

Fig 2. Six selected areas of ontology in the Intelligent Dashboard for Managers

Source: [1, p. 61].

In the years 2011-2013 we carried out four experiments with a created prototype and users participation. The results of these experiments are optimistic (discussed inter alia [12]). However, the usefulness of these solutions depends mainly on substantive content, that is, correctly building the ontology of financial or economic knowledge.

\section{DESIGN PROCESS OF FINANCIAL ONTOLOGY}

In the literature many different approaches to design of an ontology can be found (a wide review of the issue is presented in: [13]). There are many methods describing the methods of creating ontology for information systems. These are inter alia: Cyc, KBSI, TOVE, EMA, HOLSAPPLE, HCONE, System KACTUS, SENSUS, UPON, METHAONTOLOGIA, On-To-Knowledge method (a wide review of the issue is presented in: [14], [15]). But so far there is no single approach accepted by all.

Based on the analysis of existing methodologies and our research, a method of creating an ontology of financial indicators has been proposed. In this method, the following stages are distinguished (see also: [1], [6], [16], [17]):

1. Definition of the goals, scope, and constraints of the created ontology. While creating an ontology, assumptions about the created model of knowledge that will apply during its building have to be provided. That requires an answer to the question: what will the created ontology be used for?

2. Conceptualization of the ontology. Independently of the field that is to be modeled by using an ontological approach, it is the most important stage in creating a model based on ontology (see inter alia [18, p. 2036]). It includes the identification of all concepts, definition of classes and their hierarchic structures, modeling relations, identification of instances, specification of axioms, and rules of reasoning.

3. Verification of the ontology's correctness by experts. In this stage, the constructed ontology is verified by experts who did not participate in the process of conceptualization. Verification is carried out in two steps. The first concerns a formal verification of the specified ontology (e.g. incorrect relations are indicated) with the use of a given editor. The second step is carried out by experts from the given field and concerns content verification which includes verification of the correctness of topics' definitions, correctness of taxonomic topics, and correctness of relational dependences between topics. In the proposed method of building an ontology of financial knowledge verification and validation were isolated in accordance with approach used in software engineering (see [19]).

4. Encoding the ontology is described in the formal language or editor of ontology. The result of this stage is the encoded ontology. Two basic stages of encoding of ontology are: (1) entering all topics and creating a taxonomy of these topics, and (2) entering all other types of relations between topics.

5. Validation and evaluation of the built ontology. In this stage, the encoded ontology is checked to ensure it meets the needs of the managers. Validation is carried out in three areas. Firstly, validation of usefulness and correctness of the created ontology by experts (managers) who will potentially use it. Secondly, evaluation of the application with a created ontology is carried out by managers. Finally, the validation of predefined use cases is carried out. That requires an answer to the questions: will the created ontology be useful for the managers who will use it?

Figure 3 shows the design process of an ontology of financial knowledge. 


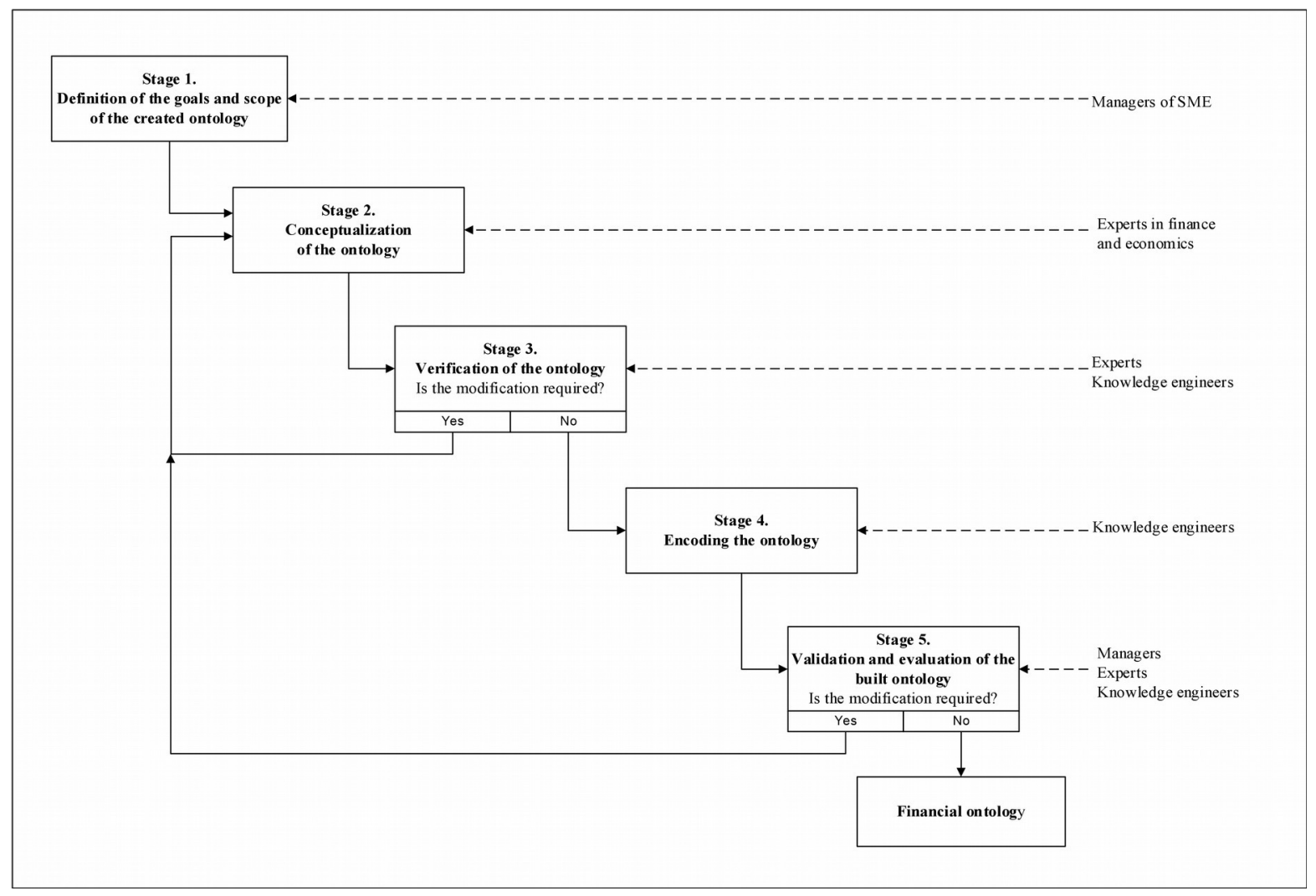

Fig. 3. Design process for an ontology of financial knowledge

Source: own elaboration.

The important stage in the described method is the conceptualization of financial indicators. The process of conceptualization of an ontology is an intellectual activity of organizing knowledge acquired from a given domain knowledge. This is carried out by the person, either an expert or in collaboration with an expert, responsible for creating the model of knowledge without the support of automated tools (see inter alia [18, p. 2036]). In the literature [14], [15]) the following phases in the conceptualization of the ontology of financial knowledge are shown (see also: [6]):

(a) Identification and definition of all topics. A topic, representing any concept, is "a syntactic construct that corresponds to the expression of a real-world in a computer system" $[10$, p. 60]. A topics' list is determined by experts in a given domain of economic knowledge. These topics include, beside their names, also their synonyms and descriptions.

(b) Creating a taxonomy of topics. Specification of taxonomic relations between distinguished topics and defining classes and subclasses. In general, these relationships describe the topics generalization. The description of a taxonomy can be presented in graphic or tabular form. An interesting approach to creating a taxonomy is proposed in METHONTOLOGIA (see i.e. [14]).

(c) Definition of all other types of relations between topics, notably the basic relationships aggregate of (Aggregate Member) was defined. Moreover, within each ontology, additional relations were defined. (d) The list of all the individual relationships existing in the ontology. The list includes: the name of the relationship, source topic, and target topic.

(e) Description of functions and rules. This description contains: name, input, output, initial and final conditions, and definition of operations.

(f) Description of usage scenarios. Usage scenarios, also called use case views, describe demonstration analyses of economic topics occurring in this ontology.

Building an ontology always denotes analysis and organizing of knowledge. That work has required multi-domain expertise, both theoretical and practical, in economics, finance, and informatics.

\section{CASE STUDY - CONCEPTUALIZATION OF FINANCIAL ONTOLOGY}

The important stage in the described method of creating an ontology is the conceptualization of ontology. We will illustrate it in the example of the analysis of Return on Sales (ROS) indicator. ROS is one of indicators that managerial staff analyses to evaluate a company's efficiency. This measure is helpful to management by providing insight into the profit structure of sales. An increasing ROS indicates that the company is growing more efficiently, while a decreasing ROS signals financial troubles. Managers often use the ROS indicator and sales analysis reports to identify market opportunities and areas where they could increase the volume of sales. 
The conceptualization of the ontology of the ROS indicator was as follows:

1. Identification and definition of all topics. Table I presents the example of the topics list.

TABLE I.

THE EXAMPLE OF TOPICS LIST

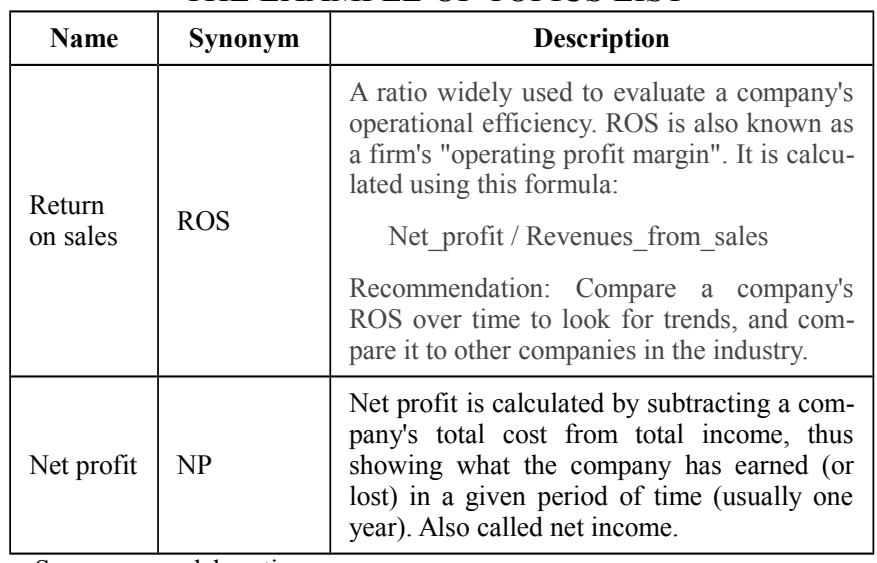

Source: own elaboration.

2. Creating a taxonomy of topics. Figure 4 shows the taxonomy for topic Indicators and topic Profitability evaluation indicators.

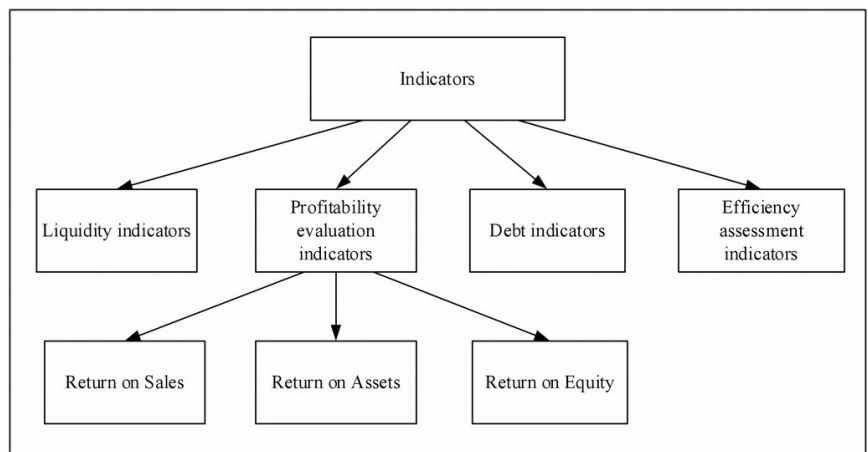

Fig. 4. Example of the taxonomy for topic Indicators and topic Profitability evaluation indicator

Source: [1, p. 64].

3. Definition of all other types of relations between topics. In this ontology, the basic relationship aggregate of (Aggregate - Member) is defined. Moreover, additional relations are defined, for example: potential growth, proportional positive/negative change, is the sum, is the quotient.

4. The list of all the user defined relationships existing in the ontology. The description of a taxonomy can be presented in graphic or tabular form. Figure 5 shows the relations existing between topic Return on Sales (in the class Profitability evaluation indicators) and topics: Net profit, and Revenues from sales ((in the class Total income). Solid lines denote taxonomic relations (relation Subclass - of), whereas broken lines denote domain-specific relations (e.g. relation is the quotient).

5. Description of functions and rules. The definition describes how to compute and interpret their values. This description can contain: name, input, output, initial and final pre-conditions, and definition of formula (see also: [9]). The following description specifies the example of the indicator Return on Sales:

Name:

Indicator Return on Sales (ROS)

\section{Input:}

Result of Net profit (NP)

type: value extracted from Balance Sheet

Revenues from sales (RS)

type: number, value extracted from Balance Sheet

\section{Output:}

Return on Sales

Description/formula:

$$
R O S=N P / R S
$$

Final conditions:

if $($ ROS $<$ value_1)

Interpretation 1

else if (value_1 $>$ ROS < value_2)

Interpretation_2

else if ....

....

else if ( $R O S>$ value_n)

Interpretation_n

6. Description of usage scenarios. Usage scenarios, also called use case views, describe demonstration analyses of economic topics occurring in this ontology. For example, a manager analyzes the ROS indicator and would like to identify causes of a decreasing value ROS:

a. The manager analyzes the semantic network, from which it follows that the Return on Sales indicator depends on two values: Net profit and Revenues from sales.

$b$. From the BI system the manager receives the values of the Net profit and Revenues from sales. It notices that the company's value of net profit is worse than for the previous period (Fig. 6).

c. The manager analyzes the semantic network of the Net profit to identify two parameters: Total income and General costs. 


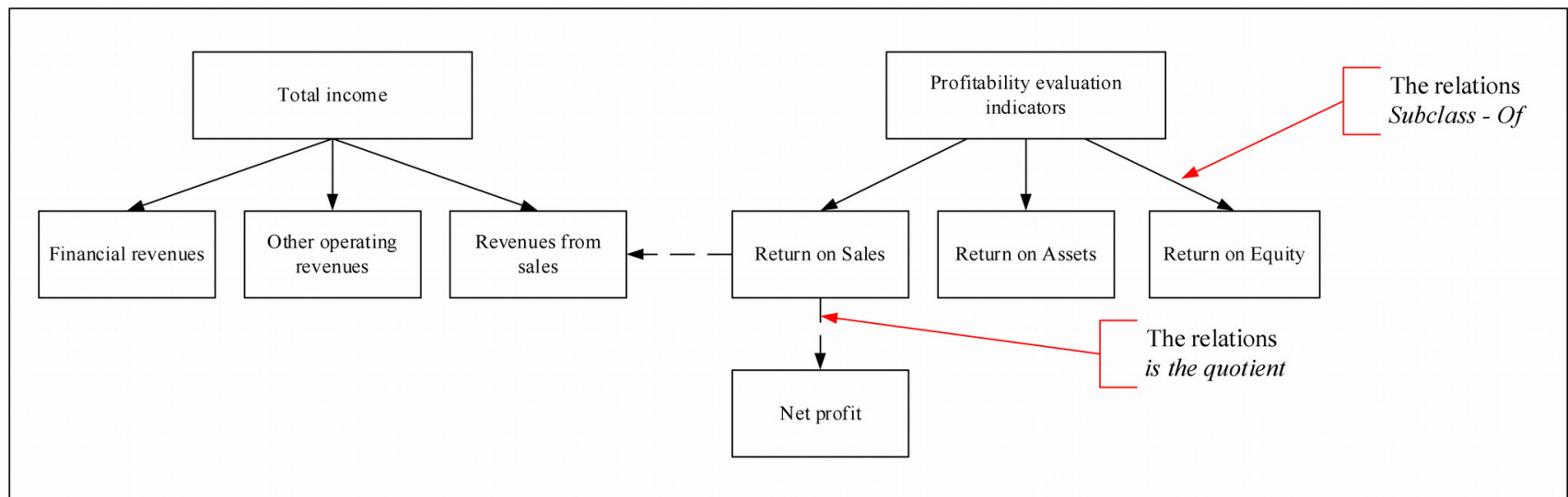

Fig. 5. The example of illustrated relationships is the quotient

\section{Source: own elaboration.}

d. From the BI system the manager receives the values of the Total income and General costs. It notices that the company's value for General costs is worse than the previous period.

$e$. The manager analyzes the semantic network of the General costs to identify causes of unfavorable values from the ROS indicator. f. Based on the analysis conducted of economic indicators, the manager can undertake corrective actions which may potentially result in improving the company's Return on Sales.

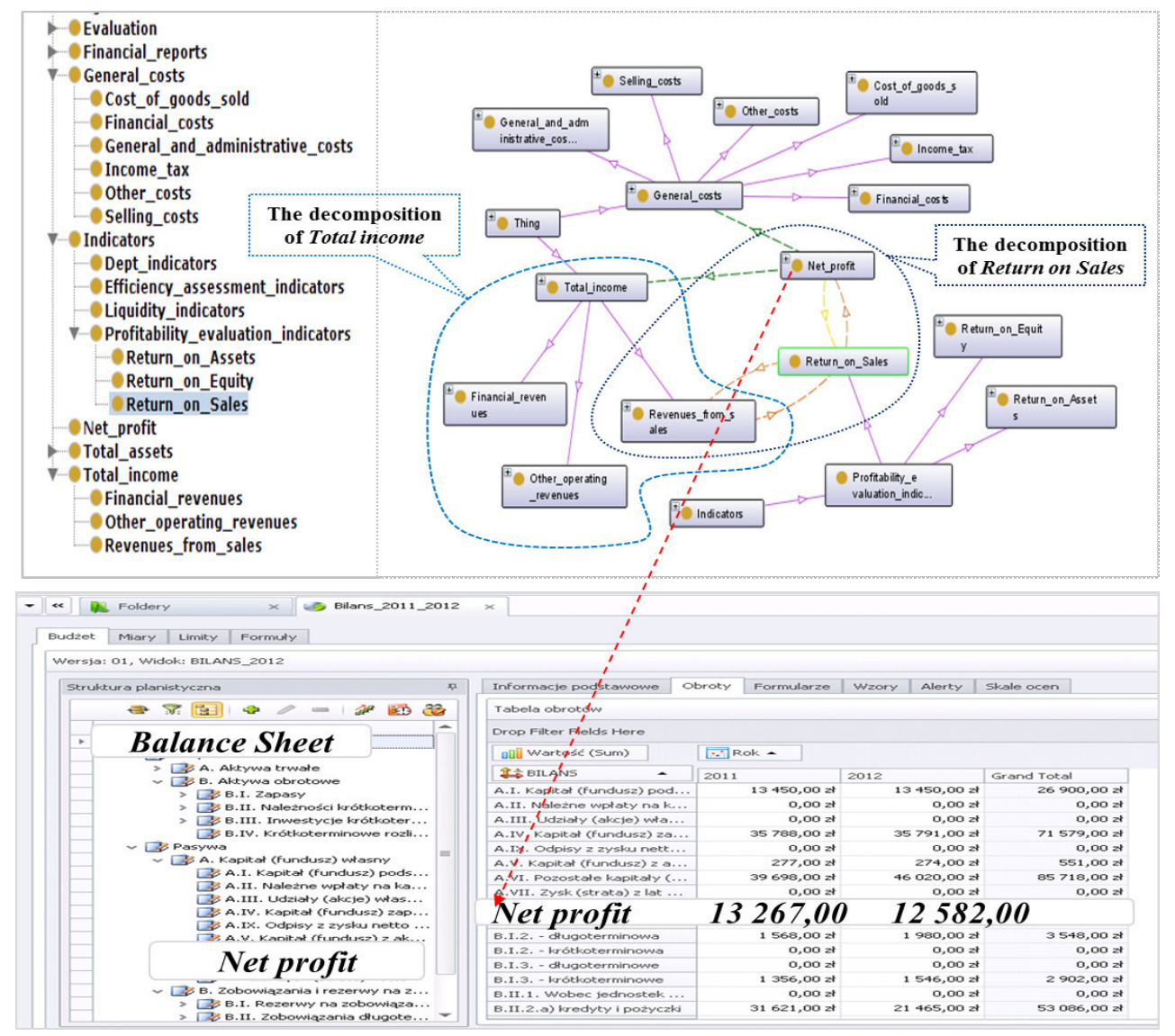

Fig. 6. . Example of visualization of entered ontology of ROS indicator and of Balance Sheet extracted from the TETA BI system 
The scenario is presented in Figure 6. The screenshot shows the expansion of the selected topic: General costs, Total income and Profitability evaluation indicators. On the diagram, it is the area encircled by a dashed line, with new topics being a subclass of the topic Total income. A semantic search is provided to avoid difficulties related to decision makers' interpretation of financial information. This gives the user the opportunity to search data sources taking into account not only structural dependences, but also the semantic context. In this figure there are two types of lines between topics: (1) the solid line represents a relation Subclass - of and (2) the dashed line represents the experts' defined relations. Business data contains a lot of hidden relationships and dependencies that make their usage difficult. To interpret the values of financial indicators correctly, many measures and ratios need to be examined that either directly or indirectly influence the final result. Explicit visualization not only makes the interpretation of indicators easier, but it also contributes to finding explanations of current values of indicators.

\section{CONCLUSION}

The use of a financial ontology seems to be a promising extension for Business Intelligence systems. It not only improves the efficiency of analysis, but also increases the capacity of understanding of financial data.

In this paper, the approach to the ontology of the financial knowledge design process was presented. The stages of ontology design were described and illustrated using the Business Intelligence system. In the case study, the topic map of key performance indicators is presented, with their structures and relationships. The financial ontology was implemented in the extended Business Intelligence system, which will be soon commercialized by TETA BI Center.

Many extensions and applications of this work are possible. Current work is directed toward the development of smart navigation throughout the very large field of ontological concepts, and the method of financial ontology updating by adding new concepts either through a SME manager or data mining modules. Suggested approach could be used in bigger enterprises too.

\section{REFERENCES}

[1] J. Korczak, H. Dudycz and M. Dyczkowski, "Specification of Financial Knowledge - Case of Intelligent Dashboard for Managers", Business Informatics, Wrocław University of Economics Research Papers, no. 2 (28), 2013, pp. 56-76.

[2] G. S. Nelson, Business Intelligence 2.0: Are we there yet?, SAS Global Forum 2010, http://support.sas.com/resources/papers/proceedings 10/040-2010.pdf.

[3] N. Raden, Business Intelligence 2.0: Simpler, More Accessible, Inevitable, February 01, 2007, http://www.informationweek.com/news/software/bi/197002610.
[4] D. Sell, L. Cabral, E. Motta, J. Domingue and R. Pacheco, Adding Semantics to Business Intelligence, 2008, http://dip.semanticweb.org/documents/WebSpaperOUV2.pdf.

[5] J. Korczak and H. Dudycz, "Approach to visualisation of financial information using topic maps", in: Information Management, B.F. Kubiak, A. Korowicki, Eds., Gdańsk: Gdańsk University Press, 2009, pp. 86-97.

[6] J. Korczak, H. Dudycz and M. Dyczkowski, "Design of Financial Knowledge in Dashboard for SME Managers", in: Proceedings of the 2013 Federated Conference on Computer Science and Information Systems. Annals of Computer Science and Information Systems, vol. 1, M. Ganzha, L. Maciaszek, M. Paprzycki, Eds. Polskie Towarzystwo Informatyczne, IEEE Computer Society Press, Warsaw, Los Alamitos, CA, 2013, pp. 1111-1118.

[7] J. Korczak, H. Dudycz and M. Dyczkowski, "Intelligent Dashboard for SME Managers. Architecture and Functions", in: Proceedings of the Federated Conference on Computer Science and Information Systems FedCSIS 2012, M. Ganzha, L. Maciaszek, M. Paprzycki, Eds., Polskie Towarzystwo Informatyczne, IEEE Computer Society Press, Warsaw, Los Alamitos, CA, 2012, pp. 1003-1007.

[8] L. Wise, The Emerging Importance of Data Visualization, part 1, October 29, 2008, http://www.dashboardinsight.com/articles/businessperformance-management/the-emerging-importance-of-data-visualization-part-1.aspx

[9] S. Smolnik and I. Erdmann, "Visual Navigation of Distributed Knowledge Structures in Groupware - Base Organizational Memories", Business Process Management Journal, vol. 9, no. 3, 2003, pp. 261280.

[10] B. L. Grant and M. Soto, "Topic maps, RDF Graphs, and ontologies visualization", in: Visualizing the Semantic Web. XML-based Internet and information visualization, second edition, V. Geroimenko, C. Chen Eds., London: Springer-Verlag, 2010, pp. 59-79.

[11] L. W. M Wienhofen, "Using Graphically Represented Ontologies for searching Content on the Semantic Web", in: Visualizing the Semantic Web. XML-Based Internet and Information Visualization, V. Geroimenko, C. Chen, Eds., London: Springer-Verlag, 2010, pp. 137153.

[12] H. Dudycz, "Heuristic evaluation of visualization in the semantic searching economic information. The Comparative Analysis of Four Experiments", Information Systems in Management, vol. 2, no. 3, 2013, pp. 194-206.

[13] B. Smith, Ontology and Information Systems, 2010, http://ontology.buffalo.edu/ontology $\% 28$ PIC $\% 29$.pdf

[14] A. Gomez-Perez, O. Corcho and M. Fernandez-Lopez, Ontological Engineering: with examples from the areas of Knowledge Management, e-Commerce and the Semantic Web, London: Springer-Verlag, 2004.

[15] F. N. Noy and D. L. McGuinness, Ontology Development 101: A Guide to Creating Your First Ontology, 2005 http://www.ksl.stanford.edu/people/dlm/papers/ontology101/ontology101-noy-mcguinness.html.

[16] H. Dudycz, The topic map as a visual representation of economic knowledge (in polish), Wrocław: Wydawnictwo Uniwersytetu Ekonomicznego we Wrocławiu, 2013.

[17] H. Dudycz, "Approach to the conceptualization of an ontology of an early warning system", in: "Information Systems in Management XI. Data Bases, Distant Learning, and Web Solutions Technologies", P. Jałowiecki, P. Łukasiewicz, A. Orłowski, Eds., Warsaw: Warsaw University of Life Sciences, Department of Informatics, 2011, pp. 2939 .

[18] M. B. Almeida and R. R. Barbarosa, "Ontologies in Knowledge Management Support: A Case Study", Journal of the American Society for Information Science and Technology, no. 10 (60), 2009, pp. 20322047.

[19] I. Sommerville, Software engineering, $9^{\text {th }}$ ed., Harlow: Addison-Wesley, 2010. 